\title{
Reliability estimation and ratio distribution in a general exponential distribution
}

\author{
Chang-Soo Lee ${ }^{1} \cdot$ Yeung-Gil Moon ${ }^{2}$ \\ ${ }^{1}$ Department of Flight Operation, Kyungwoon University \\ ${ }^{2}$ Department of Tourism Quality Management, Kangwon Tourism College \\ Received 26 February 2014, revised 17 March 2014, accepted 1 April 2014
}

\begin{abstract}
We shall consider the estimation for the parameter and the right tail probability in a general exponential distribution. We also shall consider the estimation of the reliability $P(X<Y)$ and the skewness trends of the density function of the ratio $X /(X+Y)$ for two independent general exponential variables each having different shape parameters and known scale parameter. We then shall consider the estimation of the failure rate average and the hazard function for a general exponential variable having the density function with the unknown shape and known scale parameters, and for a bivariate density induced by the general exponential density.
\end{abstract}

Keywords: Digamma function, general exponential distribution, hazard function, hazard rate, mean square error, trigamma function.

\section{Introduction}

For independent random variables $X$ and $Y$, and a real number $c$, the probability $P(X<$ $c Y$ ) is given by: (i) it is the reliability when $c=1$, (ii) it is the distribution of the ratio $X /(X+Y)$ when $c=t /(1-t)$ for $0<t<1$.

The reliability will increase the need for the industry to perform the systematic study for the identifications and the reduction for causes of the failures. These reliability studies must be performed by persons who (a) can identify and quantify for the modes of failures, (b) know how to obtain and analyze the statistics of failure occurrences, and (c) can construct mathematical models of the failure that depend on, for example, parameters of the material strength or the design quality, the fatigue or the wear resistance, and the stochastic nature of the anticipated duty cycle in Saunders (2007).

Ali et al. (2010) studied the ratio of two independent exponentiated Pareto variables. Lee and Lee (2010) considered the reliability and the ratio in a right truncated Rayleigh distribution. Lee and Lee (2012) derived the density function for the ratio of two independent weighted exponential random variables and observed the skewness of the ratio density. Yun and Lee (2012) studied estimating the reliability and the distribution of the ratio in two

\footnotetext{
${ }^{1}$ Corresponding author: Associate professor, Department of Flight Operation, Kyungwoon University, Gumi 730-850, Korea. E-mail: cslee@ikw.ac.kr

2 Associate professor, Department of Tourism Quality Management, Kangwon Tourism College, Taebaek 235-711, Korea.
} 
independent variables with different distributions. Ali et al. (2012) considered estimations of reliability in a four parameter generalized gamma distribution. Gupta and Kundu (1999) studied properties for a generalized exponential distribution, and Ali et al. (2007) introduced several generalized distributions. Kundu and Gupta (2007) reported that the generalized exponential distribution is very flexible and effective in analyzing the time data in place of gamma and Weibull models. Kundu and Pradhan (2009) estimated parameters of the generalized exponential distribution in presence of hybrid censoring, and Hussain (2011) studied the ROC curve model from the generalized exponential distribution.

In this paper, we shall consider the estimations for the parameter and the right tail probability in a general exponential distribution. We also shall consider the estimation of the reliability $P(X<Y)$ and the skewness trends of the density function of the ratio $X /(X+Y)$ for two independent general exponential variables each having different shape parameters and the known scale parameter. We then shall consider the estimation of the failure rate average and the hazard function for a general exponential variable having the density function with the unknown shape and known scale parameters, and for a bivariate density induced by the general exponential density.

\section{Estimating shape parameter and right-tail probability}

A general exponential variable $X$ has the following density function ;

$$
f(x)=\frac{\alpha}{\beta} e^{-x / \beta}\left(1-e^{-x / \beta}\right)^{\alpha-1}, x>0
$$

and its distribution function is

$$
F(x)=\left(1-e^{-x / \beta}\right)^{\alpha}, x>0,
$$

where $\alpha>0$ is the shape parameter and $\beta>0$ is the scale parameter.

The general exponential variable $X$ is considered as an alternative to the gamma and Weibull distributions for analyzing the failure time in Gupta and Kundu $(1999,2007)$.

From the density function (2.1) and the formula 5.8 in Oberhettinger and Badii (1973), we can obtain the following moment generating function $M_{X}(t)$ of $X$ :

$$
M_{X}(t)=\Gamma(\alpha+1) \frac{\Gamma(1-\beta t)}{\Gamma(\alpha+1-\beta t)}, \text { if } t<1 / \beta
$$

From the moment generating function, we can obtain

$$
E(X)=\beta(\psi(\alpha+1)-\psi(1))
$$

and

$$
E\left(X^{2}\right)=\frac{\beta^{2}}{\Gamma^{2}(\alpha+1)}\left[2\left(\Gamma^{\prime}(\alpha+1)\right)^{2}-2 \Gamma^{\prime}(\alpha+1) \Gamma(\alpha+1) \Gamma^{\prime}(1)+(\Gamma(\alpha+1))^{2} \Gamma^{\prime \prime}(1)-\Gamma^{\prime \prime}(\alpha+1) \Gamma(\alpha+1)\right],
$$

where $\Gamma^{\prime}(x)=\psi(x) \Gamma(x)$ and $\Gamma^{\prime \prime}(x)=\left[\psi^{\prime}(x)+\psi^{2}(x)\right] \Gamma(x)$ for the gamma function $\Gamma(x)$, the digamma function $\psi(x)$ and the trigamma function $\psi^{\prime}(x)$ in Abramowitz and Stegun (1970). 
Now, assume that the scale parameter $\beta$ in the density function (2.1) is known. Then, we shall consider estimations of the shape parameter $\alpha>0$ in the density function (2.1) with known $\beta=1$.

Assume $X_{1}, \cdots, X_{m}$ be a random sample from the density function (2.1) with known $\beta=1$.

Note that the complete and sufficient statistics for the shape parameter $\alpha$ in the assumed model is

$$
\sum_{i=1}^{m} \ln \left(1-e^{-X_{i}}\right)
$$

From the likelihood function of the density functions (2.1), the maximum likelihood (ML) estimator for the shape parameter $\alpha$ in the assumed model is

$$
\widehat{\alpha}=m /\left(-\sum_{i=1}^{m} \ln \left(1-e^{-X_{i}}\right)\right) .
$$

We need the following results (see Lehmann, 1983) to derive mean and variance of the ML estimator for the shape parameter $\alpha$ in (2.3).

Lemma 2.1 Let $X$ be a gamma variable with the shape parameter $\alpha$ and the scale parameter $\beta$. Then $E(1 / X)=1 /((\alpha-1) \beta)$ and $E\left(1 / X^{2}\right)=1 /\left((\alpha-1)(\alpha-2) \beta^{2}\right)$, for $\alpha>2$.

From (2.2) and the result in Hussain (2011), $-2 \alpha \sum_{i=1}^{m} \ln \left(1-e^{-X_{i}}\right)$ follows chi-square distribution with $2 m$ degree of freedom. Therefore from Lemma 2.1,

$$
E(\widehat{\alpha})=\frac{m}{m-1} \alpha \text { and } \operatorname{Var}(\widehat{\alpha})=\frac{m^{2}}{(m-1)^{2}(m-2)} \alpha^{2}, \text { for } m>2 .
$$

From the results in (2.4), an unbiased estimator $\widetilde{\alpha}$ of the shape parameter $\alpha$ is given by

$$
\widetilde{\alpha}=(m-1) /\left(-\sum_{i=1}^{m} \ln \left(1-e^{-X_{i}}\right)\right) .
$$

From the result in (2.2) and Lehmann-Scheffe Theorem in Rohatgi (1976), the unbiased estimator $\widetilde{\alpha}$ is a UMVUE of the shpae parameter $\alpha$ having variance $\frac{\alpha^{2}}{m-2}$.

From the result in (2.4) and above result, we can obtain the following result.

Proposition 2.1 Assume $X_{1}, \ldots, X_{m}$ be a random sample from the density function (2.1) with known $\beta=1$. An unbiased estimator $\widetilde{\alpha}$ performs better than the ML estimator $\hat{\alpha}$ in the sense of MSE.

We can obtain an $(1-\gamma) 100 \%$ confidence interval for the shape parameter $\alpha$ as following:

$$
\left(\frac{\chi_{\gamma / 2}^{2}(2 m)}{-2 \sum_{i=1}^{m} \ln \left(1-e^{-X_{i}}\right)}, \frac{\chi_{1-\gamma / 2}^{2}(2 m)}{-2 \sum_{i=1}^{m} \ln \left(1-e^{-X_{i}}\right)}\right)
$$


where $\chi_{\alpha}^{2}(2 m)$ is the $\alpha$-th quantile of the chi-square distribution with degree of freedom $2 m$.

From the distribution function $F(x)$ in (2.1) with known $\beta=1$, the right-tail probability of the general exponential variable $X$ is ;

$$
R(t ; \alpha) \equiv P(X>t)=1-\left(1-e^{-t}\right)^{\alpha}, \text { for } t>0 .
$$

For given $t>0$, since $\frac{d}{d \alpha} R(t ; \alpha)$ is positive, $R(t ; \alpha)$ is a monotone increasing function of $\alpha$. Therefore. the inference on $R(t ; \alpha)$ is equivalent to the inference on $\alpha$ in McCool (1991). And hence it is sufficient for us to consider estimation of $\alpha$ instead of estimating $R(t ; \alpha)$. And then we obtain the following Proposition 2.2.

Proposition 2.2 Let $R(t ; \alpha)$ be the right-tail probability of the general exponential variable $X$ having the density function (2.1) with known $\beta=1$. If $\widetilde{\alpha}$ and $\hat{\alpha}$ are the UMVUE and the ML estimator of the shape parameter $\alpha$ respectively, then $R(t ; \widetilde{\alpha})$ performs better than $R(t ; \hat{\alpha})$ in the sense of mean squared error (MSE).

Because the hazard rate, the hazard function and the hazard rate on average play an important role in the reliability in Saunders (2007), we show those definitions here :

$$
\begin{aligned}
A H(t ; \alpha) & =-\frac{1}{t} \ln R(t ; \alpha), R(t ; \alpha)=P(X>t ; \alpha): \text { hazard rate on average, } \\
h(t ; \alpha) & =f(t ; \alpha) / R(t ; \alpha), f(t ; \alpha) \text { is the density of } X: \text { hazard rate, } \\
H(t ; \alpha) & =-\ln R(t ; \alpha), \frac{d}{d t} H(t ; \alpha)=h(t ; \alpha): \text { hazard function. }
\end{aligned}
$$

Lemma 2.2 Assume $X$ be the general exponential variable having the density function (2.1) with the shape parameter $\alpha$ and the known scale parameter $\beta=1$. Then

(a) $h(t ; \alpha)$ and $H(t ; \alpha)$ are monotone decreasing functions of the shape parameter $\alpha$.

(b) $A H(t ; \alpha)$ is monotone increasing of the shape parameter $\alpha$.

Proof (a) Since $R(t ; \alpha)$ is the monotone increasing function of $\alpha, h(t ; \alpha)$ and $H(t ; \alpha)$ are monotone decreasing function of the shape parameter $\alpha$.

(b) Since $\frac{d}{d \alpha} h(t ; \alpha)=\left[\left(1-e^{-t}\right)^{-\alpha}-1\right]^{-2} e^{-t}\left(1-e^{-t}\right)^{-1} \cdot\left[\left(1-e^{t-}\right)^{-\alpha}-1-\left(1-e^{-t}\right)^{-\alpha}\right.$. $\left.\ln \left(1-e^{t-}\right)^{-\alpha}\right]$, and the sign of $r(\alpha) \equiv\left[\left(1-e^{-t}\right)^{-\alpha}-1-\left(1-e^{-t}\right)^{-\alpha} \cdot \ln \left(1-e^{-t}\right)^{-\alpha}\right]$ and the sign of $\frac{d}{d \alpha} h(t ; \alpha)$ are the same, putting $x=\left(1-e^{-t}\right)^{-\alpha}$, we have $r(\alpha)=x(1-\ln x)-1$, and hence $\frac{d}{d \alpha} r(\alpha)=\frac{d}{d x}(x-1-x \ln x) \frac{d x}{d \alpha}=(-\ln x)\left(1-e^{-t}\right)^{-\alpha}\left(-\ln \left(1-e^{-t}\right)\right) \geq 0$ for $\alpha>0$ and $t>0$. Therefore, for $t>0, \frac{d}{d \alpha} h(t ; \alpha) \geq 0$ for $\alpha>0$. This completes the proof.

From Proposition 2.1 and Lemma 2.2, by the similar manner as like the method of McCool (1991) in Proposition 2.2, we can obtain the following estimators:

Proposition 2.3 Assume $X$ be the general exponential variable having the density function (2.1) with known $\beta=1$, and let $\widetilde{\alpha}$ and $\hat{\alpha}$ be UMVUE and ML estimator of the shape parameter $\alpha$ respectively. Then

(a) $A H(t ; \tilde{\alpha})$ performs better than $A H(t ; \hat{\alpha})$ in the sense of MSE.

(b) $h(r ; \tilde{\alpha})$ performs better than $h(r ; \hat{\alpha})$ in the sense of MSE.

From the definition of the hazard function, $H(t) \equiv H(t ; \alpha)=-\ln R(t ; \alpha)$, we can obtain the property of the hazard function for the general exponential distribution in (2.1). 
Proposition 2.4 Assume $X$ be the general exponential variable having density function (2.1) with the shape parameter $\alpha$ and the known scale parameter $\beta=1$. Then

(a) the hazard function $H(t)$ is increasing and concave function of $t$.

(b) the general exponential distribution in (2.1) is decreasing hazard rate on average.

Proof (a) Since $H(t) \equiv H(t ; \alpha)=-\ln \left(1-\left(1-e^{-t}\right)^{\alpha}\right), H(0)=0, H^{\prime}(t)=\alpha e^{-t}\left(1-e^{-t}\right)^{\alpha-1}$ $\left(1-\left(1-e^{-t}\right)^{\alpha}\right)^{-1}>0$ for $t>0$, it is shown that $H(t)$ is an increasing function of $t>0$. Since $H^{\prime \prime}(t)=\alpha e^{-t}\left(1-e^{-t}\right)^{\alpha-2}\left(1-\left(1-e^{-t}\right)^{\alpha}\right)^{-2}\left[\left(1-e^{-t}\right)^{\alpha}+\alpha e^{-t}-1\right]$ and signs of $H^{\prime \prime}(t)$ and $g(t) \equiv\left(1-e^{-t}\right)^{\alpha}+\alpha e^{-t}-1$ for $t>0$ are the same, putting $x \equiv 1-e^{-t}$, we have $\frac{d}{d t} g(t)=\frac{d}{d x} g(t) \frac{d x}{d t}=\alpha\left(x^{\alpha-1}-1\right) e^{-t}=\alpha\left[\left(1-e^{-t}\right)^{\alpha-1}-1\right) e^{-t} \leq 0$, and hence $H^{\prime \prime}(t) \leq 0$. This completes the proof.

(b) It is sufficient for us to show that $-t^{-1} H(t)$ is an increasing function of $t>0$ in Saunders (2007).

$$
\begin{aligned}
\frac{d}{d t}\left(-t^{-1} H(t)\right) & =-t^{-2}\left(t H^{\prime}(t)-H(t)\right) \\
& =t^{-2}\left[\frac{\alpha t\left(1-e^{-t}\right)^{\alpha-1}}{1-\left(1-e^{-t}\right)^{\alpha}}-\ln \left(1-\left(1-e^{-t}\right)^{\alpha}\right)\right]>0, \quad t>0 .
\end{aligned}
$$

This completes the proof.

\section{Two independent general exponential variables}

\subsection{The estimation of the reliability}

In this section, we consider the case for the estimation of $P(X<Y)$ when $(X, Y)$ is a pair of two independent general exponential variables respectively.

As an application of this case $X$, representing water consumption a day in a city, is a general exponential variable and $Y$, representing the city's water supply, is another general exponential variable each having the density (2.1) with two shape parameters $\alpha$ and $\delta$ respectively. Now we consider the reliability $R(\eta)=P(X<Y)$ for $\eta \equiv \alpha / \delta$ as following:

Proposition 3.1 Assume $X$ and $Y$ be two independent general exponential variables each having density (2.1) with parameters $\alpha$ and $\delta$ respectively and both the scale parameter $\beta=1$. Then the reliability $R(\eta)=P(X<Y)=1 /(\eta+1)$ is a monotone decreasing function of $\eta \equiv \alpha / \delta$.

Since the reliability $R(\eta)$ is a monotone function of $\eta=\alpha / \delta$, the inference on $R(\eta)$ is equivalent to the inference on $\eta=\alpha / \delta$ in $\mathrm{McCool}$ (1991). And hence it is sufficient for us to consider the estimation of $\eta=\alpha / \delta$ instead of estimating $R(\eta)$.

Assume $X_{1}, \ldots, X_{m}$ and $Y_{1}, \ldots, Y_{n}$ be independent random samples from general exponential variables each having the density function (2.1) with parameters $\alpha$ and $\delta$ respective and both the scale parameter $\beta=1$.

Then ML estimators $\hat{\alpha}$ and $\hat{\delta}$ of parameters $\alpha$ and $\delta$ respectively are given by:

$$
\widehat{\alpha}=\frac{m}{-\sum_{i=1}^{m} \ln \left(1-e^{-X_{i}}\right)} \text { and } \widehat{\delta}=\frac{n}{-\sum_{i=1}^{n} \ln \left(1-e^{-Y_{i}}\right)} \text {. }
$$


And then for $\eta=\alpha / \delta$, ML estimator $\hat{\eta}$ of $\eta=\alpha / \delta$ is given by:

$$
\widehat{\eta}=\frac{m}{n} \sum_{i=1}^{n} \ln \left(1-e^{-Y_{i}}\right) / \sum_{i=1}^{m} \ln \left(1-e^{-X_{i}}\right) .
$$

From Lemma 2.1, we can obtain the expectation and the variance of $\hat{\eta}$ by:

$$
E(\widehat{\eta})=\frac{m}{m-1} \eta \text { and } \operatorname{Var}(\widehat{\eta})=\frac{m^{2}(m+n-1)}{(m-1)^{2}(m-2) n} \eta^{2} .
$$

From the expectation in (3.1), we can propose an unbiased estimator $\tilde{\eta}$ by:

$$
\widetilde{\eta}=\frac{m-1}{n} \frac{\sum_{i=1}^{n} \ln \left(1-e^{-Y_{i}}\right)}{\sum_{i=1}^{m} \ln \left(1-e^{-X_{i}}\right)} .
$$

By the similar manner as that deriving (3.1), the variance of $\tilde{\eta}$ is

$$
\operatorname{Var}(\widetilde{\eta})=\frac{m+n-1}{(m-2) n} \eta^{2} .
$$

From (3.1) and (3.2), by the similar manner as the method of McCool (1991) in Proposition 2.2, we can obtain the following:

Proposition 3.2 Assume $X_{1}, \ldots, X_{m}$ and $Y_{1}, \ldots, Y_{n}$ be independent samples from general exponential variables each having the density function (2.1) with parameters $\alpha$ and $\delta$ respectively and both the scale parameter $\beta=1$. Then for $R(\eta)=P(X<Y), R(\widetilde{\eta})$ performs better than $R(\widehat{\eta})$ in the sense of MSE.

From the definition of the $F$-distribution, we can obtain the following:

$$
\eta \frac{n}{m} \frac{\sum_{i=1}^{m} \ln \left(1-e^{-X_{i}}\right)}{\sum_{i=1}^{n} \ln \left(1-e^{-Y_{i}}\right)} \equiv \eta \cdot S
$$

where a pivot quantity $\eta \cdot S$ follows a $F$-distribution with $(2 m, 2 n)$ degrees of freedom.

From the result in $(3.3)$, an $(1-\gamma) 100 \%$ confidence interval for $\eta$ is given by:

$$
\left(\eta_{L}, \eta_{U}\right) \equiv\left(\frac{1}{F_{\gamma / 2}(2 n, 2 m)} \frac{m}{n} \frac{\sum_{i=1}^{n} \ln \left(1-e^{-Y_{i}}\right)}{\sum_{i=1}^{m} \ln \left(1-e^{-X_{i}}\right)}, \quad F_{\gamma / 2}(2 m, 2 n) \cdot \frac{m}{n} \frac{\sum_{i=1}^{n} \ln \left(1-e^{-Y_{i}}\right)}{\sum_{i=1}^{m} \ln \left(1-e^{-X_{i}}\right)}\right)
$$

where $F_{\alpha}(2 m, 2 n)$ is the $\alpha$-th quantile of the $F$-distribution with degree of freedom $(2 m, 2 n)$.

From Proposition 3.1 and (3.4), since $R(\eta)$ is monotone decreasing function of $\eta$, an $(1-\gamma) 100 \%$ confidence interval for $R(\eta)$,

$$
\left(R\left(\eta_{U}\right), R\left(\eta_{L}\right)\right)
$$

where $R(\eta)=1 /(\eta+1)$. 


\subsection{Skewness of the ratio $\mathrm{X} /(\mathrm{X}+\mathrm{Y})$}

In this section, we consider the ratio $R=X /(X+Y)$ when $X$ and $Y$ are two independent general exponential density function (2.1) each having with the shape parameters $\alpha>0$ and $\delta>0$ respectively and both the scale parameter $\beta=1$.

From the quotient density in Rohatgi (1976), the formula 1.110 in Gradshteyn and Ryzhik (1965), and formula 5.9 in Oberhettinger and Badii (1973), we can derive the quotient density function for $W \equiv Y / X$ as follows:

From the formula 1.110 in Gradshteyn and Ryzhik (1965) and the uniform convergence of series and the formula 5.9 in Oberhettinger and Badii (1973), for density functions $f_{X}(x)$ and $f_{Y}(x)$ of $X$ and $Y$ respectively,

$$
\begin{aligned}
f_{W}(w) & =\int_{0}^{\infty} x f_{Y}(w x) f_{X}(x) d x \\
& =\alpha \delta \int_{0}^{\infty} x e^{-(w+1) x}\left(1-e^{-x}\right)^{\alpha-1}\left(1-e^{-w x}\right)^{\delta-1} d x \\
& =\alpha \sum_{i=0}^{\infty}(-1)^{i}\left(\begin{array}{l}
\delta \\
i
\end{array}\right) \int_{0}^{\infty} e^{-((i+1) w+1) x} x\left(1-e^{-x}\right)^{\alpha-1} d x \\
& =\Gamma(\alpha+1) \sum_{i=0}^{\infty}(-1)^{i}\left(\begin{array}{l}
\delta \\
i
\end{array}\right) \frac{\Gamma((i+1) w+1)}{\Gamma((i+1) w+\alpha+1)}[\psi(\Gamma((i+1) w+\alpha+1)-\psi(\Gamma((i+1) w+1)]
\end{aligned}
$$

where $\psi(x)$ is the digamma function.

By $R=1 /(1+W)$ and the density (3.6) of $W$, the $k$ th moment of $R$ can not be represented by a closed form, but we can evaluate numerical values of the $k$ th moment for the ratio $R=X /(X+Y)$ when $X$ and $Y$ are two independent general exponential density (2.1) each having shape $\alpha>0$ and $\delta>0$ respectively and both scale $\beta=1$ as the following:

Table 3.1 Approximate mean, variance, and skewness for ratio $R$

\begin{tabular}{ccccc}
\hline \hline$\alpha$ & $\delta$ & mean & variance & skewness \\
\hline \multirow{4}{*}{$1 / 2$} & $1 / 4$ & .567 & .171 & -0.298 \\
& $1 / 2$ & .500 & .124 & 0.00 \\
& 1 & .364 & .094 & 0.492 \\
& 2 & .266 & .062 & 0.843 \\
& 4 & .203 & .041 & 1.074 \\
\hline \multirow{4}{*}{1} & $1 / 2$ & .629 & .096 & -0.482 \\
& 1 & .500 & .083 & 0.00 \\
& 2 & .386 & .062 & 0.339 \\
& 4 & .304 & .045 & 0.555 \\
& 6 & .269 & .037 & 0.647 \\
& $1 / 2$ & .726 & .066 & -0.874 \\
& 1 & .613 & .062 & -0.340 \\
& 2 & .500 & .052 & 0.00 \\
& 4 & .409 & .040 & 0.209 \\
& 6 & .367 & .034 & 0.296 \\
\hline \multirow{4}{*}{4} & $1 / 2$ & .789 & .046 & -1.211 \\
& 1 & .895 & .045 & -0.560 \\
& 2 & .590 & .040 & -0.214 \\
& 4 & .500 & .032 & 0.00 \\
& 6 & .455 & .028 & 0.079 \\
\hline
\end{tabular}

From Table 3.1, we can observe the following trends: 
Fact 3.1 Assume $X$ and $Y$ be two independent general exponential densities (2.1) each having with $\alpha>0$ and $\delta>0$ respectively and both scale $\beta=1$.

Then, for the ratio $R=X /(X+Y)$,

(a) the density of the ratio is symmetric about $1 / 2$ when $\alpha=\delta$.

(b) it is the right skewed when $\delta>\alpha=1 / 2,1,2,4$, but it is left skewed elsewhere.

\subsection{Property of a bivariate density based on the general exponential density}

A bivariate density based on the general exponential density (2.1) is as given in Feller (1966) by:

$$
f_{X, Y}(x, y)=\frac{\alpha}{x+y} e^{-x-y}\left(1-e^{-x-y}\right)^{\alpha-1}, \quad x>0, y>0
$$

From the bivariate density (3.7) we can obtain the following results.

Lemma 3.1 Let $(X, Y)$ have the bivariate density (3.7). Then

(a) the marginal densities of $X$ and $Y$ are equal to the following:

$$
f(x)=\alpha \sum_{i=0}^{\infty}(-1)^{i}\left(\begin{array}{c}
\alpha-1 \\
i
\end{array}\right) \Gamma(0,(i+1) x), x>0,
$$

where $\Gamma(0, x)=-E_{i}(-x)=\int_{x}^{\infty} \frac{e^{-t}}{t} d t$ is an exponential integral.

(b) $Z \equiv X+Y$ has the general exponential density (2.1).

(c) The density of $W \equiv X / Y$ is

$$
f_{W}(w)=1 /(1+w)^{2}, \quad w>0 .
$$

Proof (a) From the bivariate density (3.7),

$$
\begin{aligned}
f_{X}(x) & =\alpha \int_{0}^{\infty} \frac{1}{y+x} e^{-y-x}\left(1-e^{-x-y}\right)^{\alpha-1} d y \\
& =\alpha \int_{x}^{\infty} \frac{1}{t} e^{-t}\left(1-e^{-t}\right)^{\alpha-1} d t \\
& =\alpha \sum_{i=0}^{\infty}(-1)^{i}\left(\begin{array}{c}
\alpha-1 \\
i
\end{array}\right) \int_{x}^{\infty} t^{-1} e^{-(i+1) t} d t \\
& =\alpha \sum_{i=0}^{\infty}(-1)^{i}\left(\begin{array}{c}
\alpha-1 \\
i
\end{array}\right) \Gamma(0,(i+1) x), \text { by formula } 3.3 \text { in Oberhettinger }(1974) .
\end{aligned}
$$

In fact, $\int_{0}^{\infty} f_{X}(x) d x=1$, from formula 13.39 in Oberhettinger (1974) and binomial expansion of $(1+x)^{\alpha}$ for $|x|<1$.

(b) From the density for the sum of two random variables in Rohatgi (1976),

$$
f_{Z}(z)=\int_{0}^{z} f(x, z-x) d x=\alpha e^{-z}\left(1-e^{-z}\right)^{\alpha-1}, \quad z>0 .
$$


(c) It comes from the quotient density of two random variables in Rohatgi (1976) and formula 3.381(4) in Gradshteyn and Ryzhik (1965) from the bivariate density (3.7).

From the bivariate density (3.7) we can obtain the moment $E\left(X^{r} \cdot Y^{s}\right)$ of $X$ and $Y$.

Lemma 3.2 Let $(X, Y)$ have a bivariate density (3.7). Then

$$
E\left(X^{r} \cdot Y^{s}\right)=\alpha \Gamma(r+s+1) \sum_{i=0}^{\infty} \sum_{j=0}^{s}(-1)^{i+j}\left(\begin{array}{c}
\alpha-1 \\
i
\end{array}\right)\left(\begin{array}{c}
s \\
j
\end{array}\right)(i+1)^{-s-r-1}(j+r+1)^{-1}
$$

where $\Gamma(x)$ is the gamma function.

Proof From the density (3.7) and the formula 3.3 and 13.39 in Oberhettinger (1974),

$$
\begin{aligned}
E\left(X^{r} \cdot Y^{s}\right) & =\alpha \int_{0}^{\infty} x^{r} \int_{0}^{\infty} y^{s} \frac{1}{y+x} e^{-(y+x)}\left(1-e^{-(y+x)}\right)^{\alpha-1} d y d x \\
& =\alpha \int_{0}^{\infty} x^{r} \int_{x}^{\infty}(t-x)^{s} \frac{1}{t} e^{-} t\left(1-e^{-t}\right)^{\alpha-1} d t d x \\
& =\alpha \sum_{i=0}^{\infty} \sum_{j=0}^{s}(-1)^{i+j}\left(\begin{array}{c}
\alpha-1 \\
i
\end{array}\right)\left(\begin{array}{c}
s \\
j
\end{array}\right) \int_{0}^{\infty} x^{j+r} \int_{x}^{\infty} t^{s-j-1} e^{-(i+1) t} d t d x \\
& =\alpha \sum_{i=0}^{\infty} \sum_{j=0}^{s}(-1)^{i+j}\left(\begin{array}{c}
\alpha-1 \\
i
\end{array}\right)\left(\begin{array}{c}
s \\
j
\end{array}\right)(i+1)^{j-s} \int_{0}^{\infty} x^{j+r} \Gamma(s-j,(i+1) x) d x \\
& =\alpha \Gamma(r+s+1) \sum_{i=0}^{\infty} \sum_{j=0}^{s}(-1)^{i+j}\left(\begin{array}{c}
\alpha-1 \\
i
\end{array}\right)\left(\begin{array}{c}
s \\
j
\end{array}\right)(i+1)^{-s-r-1}(j+r+1)^{-1} .
\end{aligned}
$$

Remark 3.1 From Lemma 3.2, (a) If $s=r=1$, it is the product moment $E(X Y)$, (b) If $(r, s)=(1,0)$ and $(2,0)$, it is $E(X)$ and $E\left(X^{2}\right)$, respectively.

From Remark 3.1, we can obtain numerical values of the mean and the variance of $X$, the product moment $E(X Y)$, and the correlation coefficient between $X$ and $Y, \rho_{X, Y}$, when the shape parameter $\alpha$ varies in Table 3.2.

\begin{tabular}{ccccc}
\multicolumn{2}{c}{ Table 3.2 Numerical values of the mean, the variance of $X, E(X Y)$ and $\rho_{X, Y}$} \\
\hline \hline$\alpha$ & mean & variance & $E(X Y)$ & $\rho_{X, Y}$ \\
\hline $1 / 8$ & 0.0942 & 0.0880 & 0.0486 & 0.5412 \\
$1 / 4$ & 0.1746 & 0.1582 & 0.0947 & 0.4055 \\
$1 / 2$ & 0.3064 & 0.2662 & 0.1806 & 0.3257 \\
2 & 0.7486 & 0.5978 & 0.5813 & 0.0349 \\
2.2 & 0.7865 & 0.6263 & 0.6248 & 0.0099 \\
2.3 & 0.8044 & 0.6398 & 0.6459 & -0.0018 \\
3 & 0.9146 & 0.7248 & 0.7839 & -0.0726 \\
6 & 1.2212 & 0.9819 & 1.2438 & -0.2531 \\
8 & 1.3539 & 1.1054 & 1.4474 & -0.3218 \\
10 & 1.4584 & 1.2087 & 1.6777 & -0.3716
\end{tabular}

From Table 3.2 we can observe the following trend of $\rho_{X, Y}$ : 
Fact 3.2 Let $(X, Y)$ be a bivariate with the density (3.7) induced by the general exponential density (2.1). There exists an $\alpha_{0}\left(2.2<\alpha_{0}<2.3\right)$ such that $\rho_{X, Y}>0$ if $\alpha<\alpha_{0}$ and $\rho_{X, Y}<0$ if $\alpha>\alpha_{0}$.

For two random variables $X$ and $Y$ having the density (3.7) and a real number $c, P(Y<$ $c X)$ is the distribution of the ratio $R=Y /(X+Y)$ when $c=t /(1-t)$ for $0<t<1$ and by Lemma 3.1 (c), the ratio follows a uniform distribution over $(0,1)$. And the reliability is $1 / 2$, which corresponds to $c=1$ in $P(Y<c X)$.

Now, we consider estimates of the shape parameter $\alpha$ in the bivariate density (3.7). Assume $\left(X_{1}, Y_{1}\right), \cdots,\left(X_{n}, Y_{n}\right)$ be a random sample from the density (3.7) and define $Z_{i}=X_{i}+Y_{i}$, $i=1, \cdots, n$. Then from Lemma 3.1 (b), $Z_{1}, \cdots, Z_{n}$ are a random sample from the general exponential density (2.1) with parameter $\alpha$. From Proposition 2.1 , we can obtain that $\widetilde{\alpha}=$ $(n-1) /\left(-\sum_{i=1}^{n} \ln \left(1-e^{-Z_{i}}\right)\right)$ performs better than $\hat{\alpha}=n /\left(-\sum_{i=1}^{n} \ln \left(1-e^{-Z_{i}}\right)\right)$ in the sense of MSE.

\section{References}

Abramowitz, M. and Stegun, I. A. (1970). Handbook of mathematical functions, Dover Publications Inc., New York.

Ali, M. M., Pal, M. and Woo, J. (2007). Some exponentiated distributions. The Korean Communications in Statistics, 14, 93-109.

Ali, M. M., Pal, M. and Woo, J. (2010). On the ratio of two independent exponentiated Pareto variables. Austrian Journal of Statistics, 39, 329-340.

Ali, M. M., Pal, M. and Woo, J. (2012). Estimation of $P(Y<X)$ in a four parameter generalized gamma distribution. Austrian Journal of Statistics, 41, 197-210.

Feller, W. (1966). An introduction to probability theory and its applications II, John Wiley \& Sons, New York.

Gradshteyn, I. S. and Ryzhik, I. M. (1965). Table of integrals, series, and product, Academic Press, New York.

Gupta, R. D. and Kundu, D. (1999). Generalized exponential distribution. Australian and New Zealand Journal of Statistics, 41, 173-188.

Hussain, E. (2011). The ROC curve model from generalized-exponential distribution. Pakistan Journal of Statistics and Operation Research, 7, 323-330.

Kundu, D. and Gupta, R. D. (2007). Generalized exponential distribution. Journal of Statistical Planning and Inference, 136, 3130-3144.

Kundu, D. and Pradhn, B. (2009). Estimating the parameters of the generalized exponential distribution in presence of hybrid censoring. Communications in Statistics-Theory and Methods, 38, 2030-2041.

Lee, J. C. and Lee, C. S. (2010). Reliability and ratio in a right truncated Rayleigh distribution. Journal of the Korean Data \& Information Sciences Society, 21, 195-200.

Lee, J. C. and Lee, C. S. (2012). An approximate maximum likelihood estimator in a weight exponential distribution. Journal of the Korean Data 83 Information Sciences Society, 23, 219-225.

Lehmann, E. L. (1983). Theory of point estimation, John Wiley \& Sons, New York.

McCool, J. I. (1991). Inference on $P(X<Y)$ in the Weibull case. Communications in Statistics-Simulations, 20, 129-148.

Oberhettinger, F. (1974). Tables of Mellin transforms, Springer-Verlag, New York.

Oberhettinger, F. and Badii, L. (1973). Tables of Laplace transforms, Springer-Verlag, New York.

Rohatgi, V. K. (1976). An Introduction to probability theory and mathematical statistics, John Wiley \& Sons, New York.

Saunders, S. C. (2007). Reliability, life testing, and prediction of service lives, Springer-Verlag, New York.

Yun, S. U. and Lee, C. S. (2012). Estimating the reliability and distribution of ratio in two independent variables with different distributions. Journal of the Korean Data 8 Information Sciences Society, 23 1010-1025. 